\title{
Revealing Transition States during the Hydration of Clay Minerals
}

\author{
Tuan A. Ho*, Louise J. Criscenti, Jeffery A. Greathouse
}

Geochemistry Department, Sandia National Laboratories, Albuquerque, New Mexico 87185

*Corresponding author: taho@sandia.gov

\section{Supporting Information}

\section{Simulation Methods}

The LAMMPS package ${ }^{1}$ was used for all molecular dynamics (MD) simulations. The Na-MMT particle with two TOT (tetrahedral-octahedral-tetrahedral) layers was equilibrated with surrounding water in a simulation box of dimensions $100 \times 31.06 \times 73.62 \AA^{3}$ (Fig. S1A) at a temperature $\mathrm{T}=300 \mathrm{~K}$ and pressure $\mathrm{P}=1 \mathrm{~atm}$ (pressure was applied in the $\mathrm{z}$ direction only). The $\mathrm{x}$ and $\mathrm{y}$ dimensions of the Na-MMT particle were $53 \AA$ and $31.06 \AA$ respectively. When applying periodic boundary conditions in all directions for the simulation box, the particle becomes infinite in the $\mathrm{y}$ direction and finite in the $\mathrm{x}$ and $\mathrm{z}$ directions. Water molecules and $\mathrm{Na}^{+}$ions can exchange between the external fluid and the interlayer through the particle edges in the $\mathrm{x}$ direction. When water molecules enter the interlayer region, the Na-MMT particle can expand in the $\mathrm{z}$ direction.

To create the Na-MMT particle with edges described above, we first created a pyrophyllite particle with edges. A $31.06 \times 53.45 \times 18.66 \AA^{3}$ pyrophyllite supercell was cleaved on the $\left(\begin{array}{lll}0 & 1 & 0\end{array}\right)$ face $^{2-3}$ using Material Studio. ${ }^{4}$ The resulting broken bonds were saturated by adding $-\mathrm{OH}$ groups or $-\mathrm{H}$ atoms so that (i) each $\mathrm{Si}$ atom at the edge coordinates with $4 \mathrm{O}$ atoms and (ii) each edge $\mathrm{Al}$ atom coordinates with $5 \mathrm{O}$ atoms. At an aqueous interface, a 5-coordinated edge $\mathrm{Al}$ atom usually coordinates with a water molecule to complete its 6-fold coordination shell. ${ }^{5-6} \mathrm{Next}$, we substituted selected $\mathrm{Al}$ atoms in the octahedral sheet with $\mathrm{Mg}$ atoms to obtain a MMT particle with a Mg:Al ratio of 0.75:3.25. This substitution is random and subjected to two constraints (i) that no substitution site is adjacent to another in the octahedral sheet ${ }^{7}$ and (ii) that at the edge the $\mathrm{Mg}: \mathrm{Al}$ ratio is smaller than $0.2 .{ }^{8}$ The resulting negative charge was balanced by $\mathrm{Na}^{+}$ions randomly placed in the interlayer at the beginning of the simulation. 

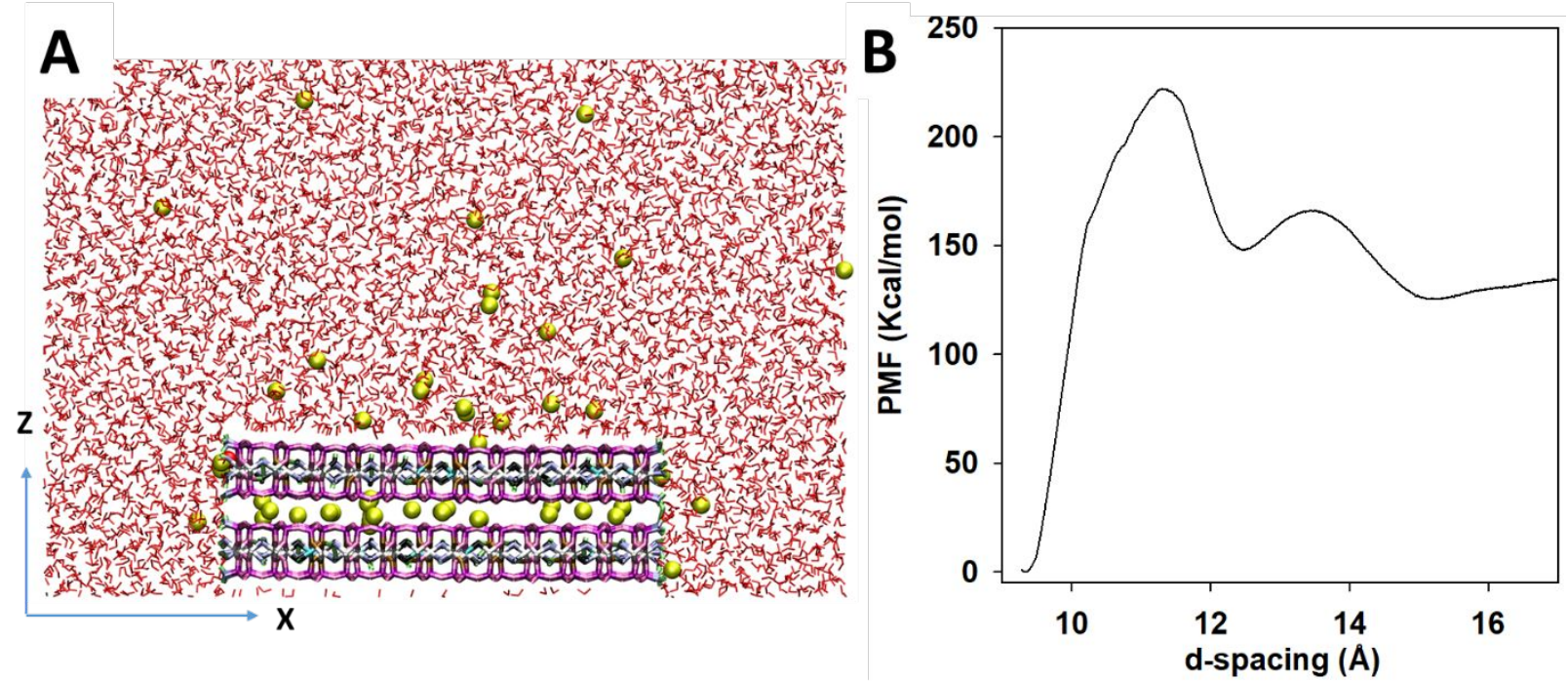

Figure S1. Simulation snapshot showing the Na-MMT particle immersed in water (A). Water molecules are depicted as red sticks and $\mathrm{Na}^{+}$ions are yellow spheres. PMF profiles calculated using ClayFF with Si-O-H and Al-O-H angle bending terms (B)

The Na-MMT particle was first simulated by the ClayFF force field ${ }^{9}$ with additional Si-O-H and Al-O-H angle terms describing edge hydroxyl groups. ${ }^{10-11}$ Water molecules were simulated using the flexible SPC water model, ${ }^{12}$ and $\mathrm{Na}^{+}$ions were modeled by the force field (FF) proposed by Dang and Smith. ${ }^{13}$ The pairwise Lennard-Jones (LJ) potential energy was expressed as: $V_{L J}=4 \varepsilon$ $\left[\left(\frac{\sigma}{r}\right)^{12}-\left(\frac{\sigma}{r}\right)^{6}\right]$, where $r$ is the distance between two atoms, $\varepsilon$ and $\sigma$ are the depth of the potential energy well and the distance at which the LJ potential is zero, respectively. LJ interactions among unlike atoms were calculated using the Lorentz-Berthelot mixing rules $\varepsilon_{i j}=\sqrt{\varepsilon_{i i} \varepsilon_{j j}}$ and $\sigma_{i j}=$ $\left(\sigma_{i i}+\sigma_{j j}\right) / 2$, unless specified in the text. Short range interactions were calculated using a cut-off distance of $10 \AA$. Long range electrostatic interactions were calculated using the PPPM (particleparticle-particle-mesh) solver. ${ }^{14}$ All simulations were performed at $300 \mathrm{~K}$ and $1 \mathrm{~atm}$ using the Nose-Hoover thermostat and barostat. ${ }^{15-16}$ A timestep of 1.0 fs was used in all simulations.

\section{FF Modification}

In a simulation box full of water (Fig. S1A) the Na-MMT particle is expected to undergo swelling (i.e., water molecules intercalate into the interlayer) as evidenced by experiment. ${ }^{17}$ Unfortunately, this did not occur in our simulation. To determine if this is a sampling problem (i.e., simulation is not long enough) or a thermodynamic limitation of the current FF, we calculated the potential of 
mean force (PMF) between two TOT layers using the COLVARS ${ }^{18}$ package in LAMMPS ${ }^{1}$. In this calculation, the umbrella sampling method was applied with a window size of $0.025 \AA$ (3.0 ns per window). The configuration of the Na-MMT particle at each window was obtained from an initial 3.0-ns steered MD simulation, ${ }^{19}$ in which the center of mass of the bottom TOT layer was kept fixed by excluding 8 atoms belonging to this layer from the integration of the equation of motion. The top TOT layer was translated away from the bottom TOT layer with a constant velocity of 5 $\AA / \mathrm{ns}$ by a harmonic spring $(k=5000 \mathrm{kcal} / \mathrm{mol})$. Note that despite these constraints each clay layer was completely flexible. The small window size (i.e., $0.025 \AA$ ) was needed to obtain sufficient overlap among windows for the convergence of the PMF calculation. The Weighted Histogram Analysis Method (WHAM) was applied to extract the PMF profile from the simulation trajectory. ${ }^{20}$ The PMF for the hydration process of the Na-MMT particle simulated by ClayFF is reported in Fig. S1B.

We observed two minima at d-spacings of $12.17 \AA$ and $15.00 \AA$, corresponding to the one-layer (1W) and two-layer (2W) hydration states of Na-MMT, respectively. ${ }^{21}$ Unfortunately, neither of these minima is global as the dry (0W) state (d-spacing $\sim 9.3 \AA$ ) has the lowest energy in the PMF profile. This would appear to be a nonphysical result since the Na-MMT particle naturally expands to the $2 \mathrm{~W}$ state under aqueous conditions. ${ }^{17}$ ClayFF successfully predicts the energetics and structural properties of the $1 \mathrm{~W}$ and $2 \mathrm{~W}$ states of Na-MMT. ${ }^{22}$ However, it does not predict well the 0W-1W hydration process. Others have used a similar layer-layer harmonic constraint to investigate the interaction between adjacent TOT layers with a d-spacing larger than $1.5 \mathrm{~nm} .{ }^{23-25}$ However, to our knowledge the $0 \mathrm{~W}-1 \mathrm{~W}$ transition has not been studied. Our simulations indicate that FF modification is needed to adequately describe this transition.

Previous ClayFF simulation studies of Na-MMT have noted the accumulation of $\mathrm{Na}^{+}$ions unrealistically deep into the hexagonal cavities created by surface bridging $\mathrm{O}$ atoms. ${ }^{26-27}$ Ferrage et al. ${ }^{28}$ modified the bridging $\mathrm{O}$ atom parameters in ClayFF to reproduce the XRD diffraction of Na-saponite. Subsequently, Szczerba et al. ${ }^{29}$ modified the parameters for the surface bridging $\mathrm{O}$ atoms near tetrahedral substitution sites, along with the parameters for tetrahedral Si and Al atoms. When applying this modification, our Na-MMT particle was not stable during an unconstrained MD simulation (i.e., distortion started at the edge $\mathrm{SiO}_{4}$ tetrahedra or $\mathrm{AlO}_{6}$ octahedra). This might 
be because the angle bending term we use to describe edges was developed in conjunction with the original version of ClayFF ${ }^{10-11}$ rather than the modified parameters used by Szczerba et al. ${ }^{29}$

There may be many ways to adjust interaction parameters among atoms in the system of Fig. S1A to obtain a reasonable PMF profile. However, we want to preserve as many interatomic potentials as possible to avoid creating an unstable Na-MMT particle structure, like the one we observed above when we applied the modification by Szczerba et al. ${ }^{29} \mathrm{We}$ also do not want to change the MMT-water pairwise interactions because the $\mathrm{O}$ parameters in ClayFF were designed for consistency with the SPC water model. ${ }^{12}$

Table S1. LJ parameters for the interaction between $\mathrm{Na}^{+}$ions and bridging $\mathrm{O}$ atoms $(o b$ and obos ClayFF types ${ }^{9}$ )

\begin{tabular}{|l|l|l|}
\hline Simulation/FF & $\boldsymbol{\sigma}(\boldsymbol{\AA})$ & $\boldsymbol{\varepsilon}(\mathbf{k c a l} / \mathbf{m o l})$ \\
\hline ClayFF $^{9}$ & 2.75776 & 0.1422 \\
\hline swell-ClayFF_1 & 2.86774 & 0.4266 \\
\hline swell-ClayFF_2 & 2.95000 & 0.4266 \\
\hline swell-ClayFF & 2.95000 & 0.4500 \\
\hline
\end{tabular}

From the PMF profile (Fig. S1B) and the previous reports of unusually strong Na-surface interactions, we considered that ClayFF overpredicts the interaction between $\mathrm{Na}^{+}$ions and surface $\mathrm{O}$ atoms, making the Na-MMT dry state too stable in water. To overcome this difficulty to obtain a reasonable thermodynamic result for the Na-MMT hydration process, we modified the LJ interaction between $\mathrm{Na}^{+}$ions and bridging $\mathrm{O}$ atoms of the MMT structure to obtain stronger repulsive forces at short distances, resulting in a lower energy barrier for interlayer hydration and swelling of the Na-MMT particle. This minor parameter adjustment does not affect the stability of the TOT structure.

In Table S1, we report three sets of modified parameters for interactions between $\mathrm{Na}^{+}$ions and bridging $\mathrm{O}$ atoms. In Fig. S2A we show the LJ potential energy as a function of the distance between a $\mathrm{Na}^{+}$ion and a surface $\mathrm{O}$ atom for each modification. Note that the swell-ClayFF_1 and swell-ClayFF_2 FFs serve as a sensitivity analysis of how the $\mathrm{Na}^{+}-\mathrm{O}_{\text {bridging }} \mathrm{LJ}$ interaction affects the PMF profile. Swell-ClayFF is the final parameter set that was validated using the structural 
properties of the dry Na-MMT model (discussed in detail below). As shown in Fig. S2A, the LJ profiles become steeper (e.g. more repulsive) at smaller distances (i.e., less than $3 \AA$ ) when changing the LJ parameters from ClayFF to swell-ClayFF_1, swell-ClayFF_2, and swell-ClayFF. The PMF profiles for the modified FFs reported in Fig. S2B indicates an improvement in the thermodynamics of the hydration process, compared to the original ClayFF (Fig. S1B). For example, for swell-ClayFF_1, the global minimum is the $2 \mathrm{~W}$ state. However, the $0 \mathrm{~W}$ state still has lower energy compared to the $1 \mathrm{~W}$ state. For swell-ClayFF_2 and swell-ClayFF, the $0 \mathrm{~W}$ state has a higher free energy compared to the $1 \mathrm{~W}$ and $2 \mathrm{~W}$ states, as expected for the hydration of Na-MMT. Comparison of the PMF profiles in Fig. S2B also illustrates how a subtle change in molecular interactions affects the thermodynamic properties of the hydration process.
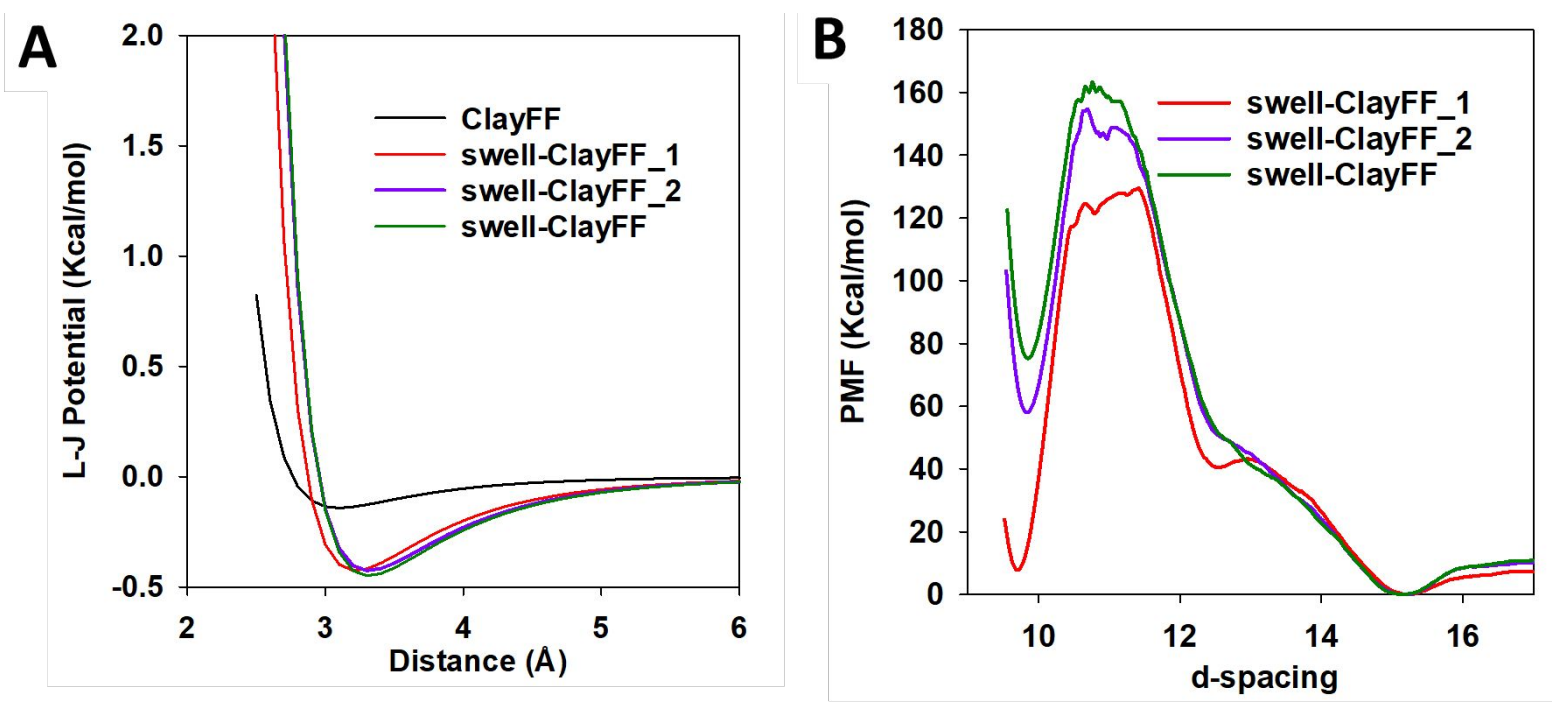

Figure S2. Pairwise LJ potential between $\mathrm{Na}^{+}$ion and bridging $\mathrm{O}$ atom calculated using different FFs (A). PMF profiles of the hydration process calculated using different variants of swell-ClayFF (B).

We now discuss structural properties of the dry Na-MMT particle simulated by ClayFF and swellClayFF to validate our LJ parameter modification. The simulation snapshot (Fig. S3A) reveals that when using ClayFF, $\mathrm{Na}^{+}$ions accumulate deep into the hexagonal cavities created by surface $\mathrm{O}$ atoms. The corresponding density profile in Fig. S3C indicates that there are two distinguishable peaks of $\mathrm{Na}^{+}$ions in the dry interlayer, and these peaks are close to those for surface $\mathrm{O}$ atoms. For swell-ClayFF, $\mathrm{Na}^{+}$ions reside at the center of the interlayer (Fig. S3B) and the density profile indicates that the $\mathrm{Na}^{+}$peak spans about $1 \AA$ (Fig. S3D). The d-spacing of dry Na-MMT calculated using swell-ClayFF is $9.81 \AA$, in better agreement with experimental results $(9.6-9.8 \AA)^{30-34}$ than 
the ClayFF result (i.e., $\sim 9.37 \AA$ in this work and Teich-McGoldrick et al. ${ }^{22}$ ). In Fig. S3E, we report the radial distribution function (RDF) between $\mathrm{Na}^{+}$ions and surface $\mathrm{O}$ atoms. For ClayFF, the first peak indicates that the distance from $\mathrm{Na}^{+}$ions to surface $\mathrm{O}$ atoms can be from 2.2 to $3.5 \AA$. For swell-ClayFF, this range increases $(2.4-4.0 \AA)$. A corresponding range of distances $(2.4-3.9 \AA)$ was obtained from static density functional theory calculations. ${ }^{35}$ Therefore, we implemented swell-ClayFF because it best reproduces the distance between $\mathrm{Na}^{+}$ions and surface $\mathrm{O}$ atoms.
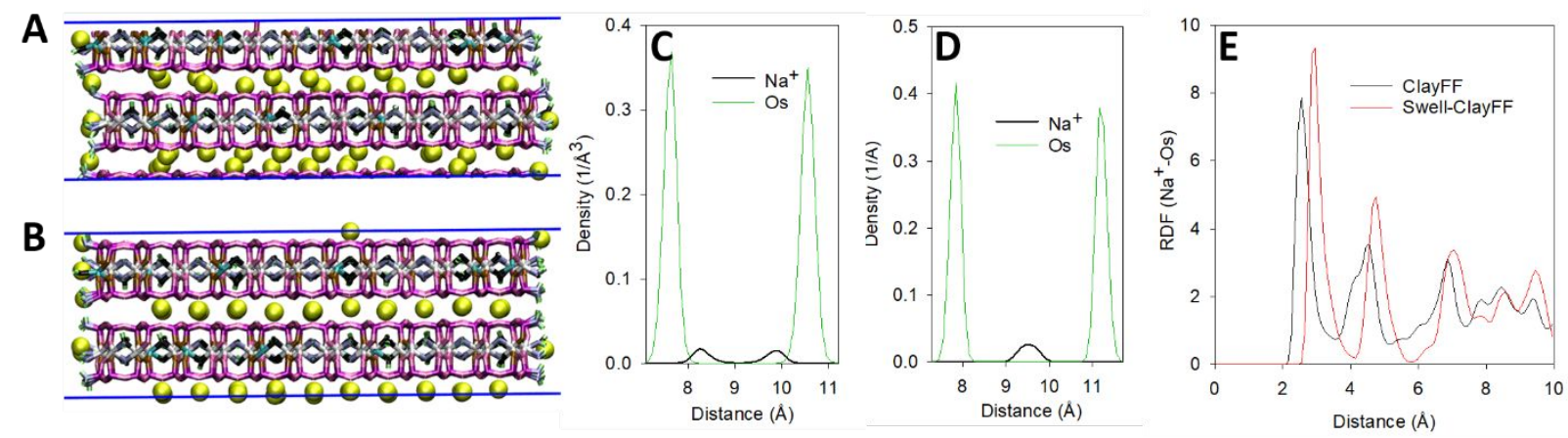

Figure S3. Simulation snapshot demonstrates the dry state of the Na-MMT particle simulated by ClayFF (A) and swell-ClayFF (B). Density profiles of $\mathrm{Na}^{+}$ions (black lines) and surface bridging $\mathrm{O}$ atoms (green lines) simulated by ClayFF (C) and swell-ClayFF (D). RDFs of $\mathrm{Na}^{+}$ions and surface $\mathrm{O}$ atoms simulated by ClayFF (black line) and swell-ClayFF (red line) (E). Atoms are colored as in Fig. S1.

In summary, by modifying the $\mathrm{Na}^{+}$-surface $\mathrm{O} \mathrm{LJ}$ potential for ClayFF, we have changed the calculated energy of the dry Na-MMT particle, which now exhibits a higher free energy than the $1 \mathrm{~W}$ and $2 \mathrm{~W}$ states as we would anticipate from experimental observations of clay swelling in water. In addition, this modification has improved the calculated d-spacing for dry Na-MMT.

\section{References}

(1) Plimpton, S., Fast Parallel Algorithms for Short-Range Molecular-Dynamics. J. Comput. Phys. 1995, 117, 1-19.

(2) Lavikainen, L. P.; Hirvi, J. T.; Kasa, S.; Schatz, T.; Pakkanen, T. A., Stability of Dioctahedral 2:1 Phyllosilicate Edge Structures Based on Pyrophyllite Models. Theor. Chem. Acc. 2015, 134, 112.

(3) Harvey, J. A.; Johnston, C. T.; Criscenti, L. J.; Greathouse, J. A., Distinguishing between Bulk and Edge Hydroxyl Vibrational Properties of 2: 1 Phyllosilicates Via Deuteration. Chemical Communications 2019, 55, 3453-3456.

(4) Accelrys, Materials Studio, Release 5.5. Accelrys Software Inc. 2010, San Diego, CA.

(5) Ho, T. A.; Greathous, J. A.; Lee, A. S.; Criscenti, L. J., Enhanced Ion Adsorption on Mineral Nanoparticles. Langmuir 2018, 34, 5926-5934. 
(6) Ho, T. A.; Greathouse, J. A.; Wang, Y. F.; Criscenti, L. J., Atomistic Structure of Mineral Nano-Aggregates from Simulated Compaction and Dewatering. Sci. Rep. 2017, 7, 15286.

(7) Loewenstein, W., The Distribution of Aluminum in the Tetrahedra of Silicates and Aluminates. Am. Miner. 1954, 39, 92-96.

(8) Newton, A. G.; Kwon, K. D.; Cheong, D. K., Edge Structure of Montmorillonite from Atomistic Simulations. Minerals 2016.

(9) Cygan, R. T.; Liang, J. J.; Kalinichev, A. G., Molecular Models of Hydroxide, Oxyhydroxide, and Clay Phases and the Development of a General Force Field. J. Phys. Chem. B 2004, 108, 1255-1266.

(10) Pouvreau, M.; Greathouse, J. A.; Cygan, R. T.; Kalinichev, A. G., Structure of Hydrated Gibbsite and Brucite Edge Surfaces: Dft Results and Further Development of the Clayff Classical Force Field with Metal-O-H Angle Bending Terms. J. Phys. Chem. C 2017, 121, 14757-14771.

(11) Pouvreau, M.; Greathouse, J. A.; Cygan, R. T.; Kalinichev, A. G., Structure of Hydrated Kaolinite Edge Surfaces: Dft Results and Further Development of the Clayff Classical Force Field with Metal-O-H Angle Bending Terms. J. Phys. Chem. C 2019, 1162811638.

(12) Teleman, O.; Jonsson, B.; Engstrom, S., A Molecular-Dynamics Simulation of a Water Model with Intramolecular Degrees of Freedom. Mol. Phys. 1987, 60, 193-203.

(13) Smith, D. E.; Dang, L. X., Computer-Simulations of Nacl Association in Polarizable Water. J. Chem. Phys. 1994, 100, 3757-3766.

(14) Hockney, R. W.; Eastwood, J. W., Computer Simulation Using Particles; Taylor \& Francis Group, LLC.: New York, 1988, p 564.

(15) Nose, S., A Molecular-Dynamics Method for Simulations in the Canonical Ensemble. Mol. Phys. 1984, 52, 255-268.

(16) Martyna, G. J.; Tobias, D. J.; Klein, M. L., Constant-Pressure Molecular-Dynamics Algorithms. J. Chem. Phys. 1994, 101, 4177-4189.

(17) Morodome, S.; Kawamura, K., Swelling Behavior of Na- and Ca-Montmorillonite up to 150 Degrees C by in Situ X-Ray Diffraction Experiments. Clays Clay Miner. 2009, 57, 150-160.

(18) Fiorin, G.; Klein, M. L.; Henin, J., Using Collective Variables to Drive Molecular Dynamics Simulations. Mol. Phys. 2013, 111, 3345-3362.

(19) Izrailev; Stepaniants; Isralewitz; Kosztin; Lu; Molnar; Wriggers; Schulten, Computational Molecular Dynamics: Challenges, Methods, Ideas. In Lecture Notes in Computational Science and Engineering, Springer-Verlag: Berlin, 1998; Vol. 4, pp 3965.

(20) Grossfield, A., Wham: The Weighted Histogram Analysis Method. http://membrane.urmc.rochester.edu/wordpress/?page id $=126$.

(21) Sposito, G., The Surface Chemistry of Soils; Oxford University Press: New York, 1984.

(22) Teich-McGoldrick, S. L.; Greathouse, J. A.; Jove-Colon, C. F.; Cygan, R. T., Swelling Properties of Montmorillonite and Beidellite Clay Minerals from Molecular Simulation: Comparison of Temperature, Interlayer Cation, and Charge Location Effects. J. Phys. Chem. C 2015, 119, 20880-20891.

(23) Sun, L.; Hirvi, J. T.; Schatz, T.; Kasa, S.; Pakkanen, T. A., Estimation of Montmorillonite Swelling Pressure: A Molecular Dynamics Approach. J. Phys. Chem. C 2015, 119, 19863-19868. 
(24) Sun, L. L.; Ling, C. Y.; Lavikainen, L. P.; Hirvi, J. T.; Kasa, S.; Pakkanen, T. A., Influence of Layer Charge and Charge Location on the Swelling Pressure of Dioctahedral Smectites. Chem. Phys. 2016, 473, 40-45.

(25) Hsiao, Y. W.; Hedstrom, M., Swelling Pressure in Systems with Na-Montmorillonite and Neutral Surfaces: A Molecular Dynamics Study. J. Phys. Chem. C 2017, 121, 2641426423.

(26) Lammers, L. N.; Bourg, I. C.; Okumura, M.; Kolluri, K.; Sposito, G.; Machida, M., Molecular Dynamics Simulations of Cesium Adsorption on Illite Nanoparticles. $J$. Colloid Interface Sci. 2017, 490, 608-620.

(27) Zaunbrecher, L. K.; Cygan, R. T.; Elliott, W. C., Molecular Models of Cesium and Rubidium Adsorption on Weathered Micaceous Minerals. J. Phys. Chem. A 2015, 119, 5691-5700.

(28) Ferrage, E.; Sakharov, B. A.; Michot, L. J.; Delville, A.; Bauer, A.; Lanson, B.; Grangeon, S.; Frapper, G.; Jiménez-Ruiz, M.; Cuello, G. J., Hydration Properties and Interlayer Organization of Water and Ions in Synthetic Na-Smectite with Tetrahedral Layer Charge. Part 2. Toward a Precise Coupling between Molecular Simulations and Diffraction Data. J. Phys. Chem. C 2011, 115, 1867-1881.

(29) Szczerba, M.; Kuligiewicz, A.; Derkowski, A.; Gionis, V.; Chryssikos, G. D.; Kalinichev, A. G., Structure and Dynamics of Water-Smectite Interfaces: Hydrogen Bonding and the Origin of the Sharp O-D-W/O-H-W Infrared Band from Molecular Simulations. Clays Clay Miner. 2016, 64, 452-471.

(30) Mooney, R. W.; Keenan, A. G.; Wood, L. A., Adsorption of Water Vapor by Montmorillonite .2. Effect of Exchangeable Ions and Lattice Swelling as Measured by XRay Diffraction. J. Am. Chem. Soc. 1952, 74, 1371-1374.

(31) Berend, I.; Cases, J. M.; Francois, M.; Uriot, J. P.; Michot, L.; Masion, A.; Thomas, F., Mechanism of Adsorption and Desorption of Water-Vapor by Homoionic Montmorillonites .2. The $\mathrm{Li}^{+}, \mathrm{Na}^{+}, \mathrm{K}^{+}, \mathrm{Rb}^{+}$and $\mathrm{Cs}^{+}$-Exchanged Forms. Clays Clay Miner. 1995, 43, 324-336.

(32) Chiou, C. T.; Rutherford, D. W., Effects of Exchanged Cation and Layer Charge on the Sorption of Water and Egme Vapors on Montmorillonite Clays. Clays Clay Miner. 1997, 45, 867-880.

(33) Rutherford, D. W.; Chiou, C. T.; Eberl, D. D., Effects of Exchanged Cation on the Microporosity of Montmorillonite. Clays Clay Miner. 1997, 45, 534-543.

(34) Calvet, R., Hydration of Montmorillonite and Diffusion of Exchangeable Cations .1. Hydration of Montmorillonite Saturated by Monovalent Cations. Ann Agron 1973, 24, 77-133.

(35) Berghout, A.; Tunega, D.; Zaoui, A., Density Functional Theory (Dft) Study of the Hydration Steps of $\mathrm{Na}+/ \mathrm{Mg} 2+/ \mathrm{Ca} 2+/ \mathrm{Sr} 2+/ \mathrm{Ba} 2+-$ Exchanged Montmorillonites. Clays Clay Miner. 2010, 58, 174-187. 